\title{
Der Unterschied zwischen dem Nicht-Tun und dem Nichts-Tun
}

\section{Christoph Bosshard}

Dr. med., Vizepräsident der FMH, Departementsverantwortlicher Daten, Demographie und Qualität

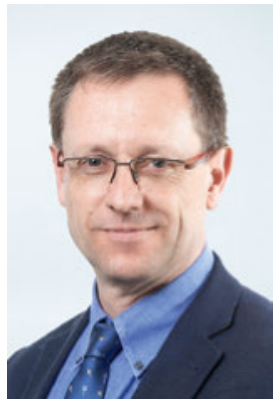

Als ich vor zirka 20 Jahren im Rahmen eines Fellowships einen handchirurgischen Ordinarius in seiner Sprechstunde begleiten durfte, konnte ich erleben, wie er mit einem nicht nur medizinisch komplexen, sondern auch ungeduldigen Patienten umging. Er verordnete ein MRI. Ich habe mir dann die Frage erlaubt, weshalb er dies getan hätte, ich könne die Indikation zu dieser Diagnostik nicht nachvollziehen. Er antwortete mir mit einem Lächeln: «Die medizinische Indikation gibt es auch nicht, aber bis zum MRI-Termin vergehen mindestens sechs bis acht Wochen, und der Patient ist ansonsten nicht gewillt, zwischenzeitlich den therapeutischen Weg weiterzugehen, welcher ihm Erfolg bringen wird.» Immer wieder denke ich an diese Situation zurück. Ich weiss leider nicht, ob das MRI wie beabsichtigt überflüssig wurde. Aber ich habe gelernt, dass es sehr viel mehr Überzeugungsarbeit braucht und damit Zeit kostet, unsere Patienten im Aushalten und Akzeptieren einer Situation zu begleiten, als eben etwas zu tun, zu handeln. Und die Zeit, ihn damit vertraut zu machen und zu unterstützen, fehlt zunehmend überall. Damit verbunden finden sich sofort diverse weitere Gründe, dem Aktivismus zu verfallen: Niemand will sich etwas zu Schulden kommen lassen, was später als Unterlassung ausgelegt werden könnte. Man will alles versucht haben! Zeit ist Geld, und das Fehlen am Arbeitsplatz auch ein medizinisches Problem. Heute bleiben Patienten nur wenige Tage im Spital, während sie früher mit gleicher Diagnose mehrere Wochen dort gewesen wären. Diese Verdichtung lässt nicht viel Zeit. Wenn ich früher meinen ärztlichen Lehrern gegenüber noch rechtfertigen musste, weshalb ich die eine oder andere diagnostische Massnahme angeordnet hatte, so höre ich heute, dass sich trotz DRG die jüngeren Kollegen in Weiterbildung rechtfertigen müssen, wenn sie etwas NICHT getan haben. Diese Angst der Unterlassung ist auch Teil unserer zunehmenden Misstrauenskultur, aufgrund welcher immer mehr Ressourcen in die Administration fliessen, in die Dokumentation und mitunter auch in medizinische Massnahmen, welche letztlich nur der Absicherung dieser Dokumentation dienen und nicht der Absicherung der Diagnose. Und doch verlangt das Gesetz überall WZW-Kriterien - oder eben doch nicht überall?
Wenn wir im Rahmen der Sozialversicherungen über neue Techniken oder auch bestehende Techniken im Vergleich zu neuen diskutieren, ist auch deren Wirksamkeit, Zweckmässigkeit und Wirtschaftlichkeit zu diskutieren. Um bezüglich Choosing Wisely oder Smarter Medicine eine Übersicht über die wissenschaftlichen Grundlagen zu erhalten und darauf aufbauend die Diskussion weiterführen zu können, veröffentlicht die FMH in dieser Ärztezeitung ein Grundlagenpapier sowie eine standespolitische Positionierung des FMH-

Es braucht viel mehr Überzeugungsarbeit und Zeit, Patienten im Aushalten und Akzeptieren einer Situation zu begleiten, als etwas zu tun.

Zentralvorstands mit klaren Forderungen. Dies unterstreicht, dass die Ärzteschaft sich auch dieser Herausforderung annimmt, wie sie es bereits mit den Thematiken Overuse [1], Guidelines [2] und Shared Decision Making [3] getan hat. Das Nicht-Tun braucht neben einer klaren medizinischen Evidenz auch Überzeugungsarbeit bei der Ärzteschaft, die Akzeptanz der Patienten und schliesslich auch der ganzen Gesellschaft, welche immer wieder 'Handeln' fordert. Dies wird uns nur gelingen, wenn auch dieser Prozess ärztlich geführt in einem administrationsentlasteten Vertrauensverhältnis der Arzt-Patienten-Beziehung seinen Platz finden kann, wo Freiräume bestehen, begründet sowohl von ChoosingWisely-Listen wie auch von Guidelines abzuweichen. Denn beide sind primär morbiditätszentriert und werden der Komplexität unserer zunehmend polymorbiden Patienten nicht genügend gerecht. Somit sollen uns diese Empfehlungen im einfachen Regelfall unterstützen, um unsere ärztliche Schaffenskraft in die komplexen Fälle investieren zu können, wo nebst der ganzen Evidenz unsere Erfahrung und Kenntnis des Einzelfalls weit über die Medizin hinaus gefordert ist.

\section{Literatur}

1 Zentralvorstand der FMH. Die Position der FMH. Overuse min dern, Behandlungsqualität steigern. Schweizerische Ärztezeitung. 2016;97(7):244-45.

2 FMH/DDQ. Die Meinung der FMH. Medizinische Guidelines: Voraussetzungen und Anwendung. Schweizerische Ärztezeitung. 2014;95(3):52-3.

3 FMH/DDQ. Die Meinung der FMH. Shared Decision Making. Schweizerische Ärztezeitung. 2014;95(50):1890-91. 